\title{
Land Use and Climate Impacts on Fluvial Systems during the Period of Agriculture (LUCIFS)
}

Cumulative Global Change is occurring by the removal of forests, conversion to cultivation of marginal land, and intensification of cultivation. Systemic Global Change, in the form of climate change and atmospheric chemistry change, is likely to alter land use patterns during the next century. All of these changes will affect rivers and their catchments, altering flows of water, sediment, nutrients, carbon and pollutants. Past changes of land and climate are still being felt in many catchments, and are difficult to understand without a historical perspective. Future changes, when superimposed on changes triggered in the past, will produce complex responses which will be difficult to anticipate.

There is therefore a clear need for a better understanding, and a better theory, of fluvial system response to land use and climate change, to anticipate and perhaps predict future changes, and to understand current dynamics. While most scientific research has been conducted in small catchments, most interest in future responses is in large catchments. The time period over which a large catchment responds to land use or climate change is much longer than is the case for a small catchment; much longer than most instrumental time series. Therefore, the paleoenvironmental record provides a unique data repository of catchment responses, fully realised over decades to centuries. This repository, or library, is an unparalleled set of experiments already carried out. Our task is to exploit this library.

The PAGES-LUCIFS Project aims to assemble a library of fluvial system responses from around the world, using case studies selected to represent both the modern array of land use and catchment types, and the areas where land use has evolved slowly over millennia and where industrial agriculture has been transplanted to non-agricultural landscapes during the last few centuries. Modelling of this library of responses is being developed to provide the tools for anticipation and/or prediction of future change.

While Global Change impacts on fluvial systems are important, change to these systems will also affect the coastal zone by increasing the flow of materials to the coast, in some cases, and decreasing flows in other cases. The changed transport of carbon and nutrients by rivers also have a role in global biogeochemistry, particularly in the coastal zone.

Rivers therefore play a role as one of the links between the major global biogeochemical systems, but, more than that, they are crucially important to human well being and for many aquatic organisms. The importance of fluvial systems, as phenomena that are affected by Global Change and affect Global Change, is clear, along with their enormous societal and ecological significance.

The LUCIFS planning group has identified five key questions to guide research, as follows:

1. What is the sensitivity to climatic change of the spatial distribution of sediment, $\mathrm{P}$ and $\mathrm{C}$ fluxes in different climate/vegetation regions?

2. How do sediment, $\mathrm{P}$, and $\mathrm{C}$ flux sensitivities to land use under climatic shifts reflect stages of land use history?

3. How are fluvial system response sensitivities under various land uses influenced by the direction of climatic change?

4. What are the thresholds and response and recovery of fluvial systems for different combinations of land use and climatic change?

5. How do engineering and other human-related modifications, including dams and reservoirs, levees, channel morphology alteration, and wetland drainage enhance or suppress climatic impacts on sediment, $\mathrm{P}$, and $\mathrm{C}$ fluxes in various climate/ vegetation regions?

In summary, these questions revolve around the sensitivity of fluvial systems to land use and climate change, both individually and in concert. To answer these questions globally, case studies from various parts of the world are needed.

A LUCIFS workshop is being planned for January 1999. The workshop will have the following purposes:

Review progress to date by each group presenting its scientific results and plans for the future.

1. Review progress in modelling

2. Assemble major conclusions that can be reached at this stage in the project, possibly to produce a multi-authored paper for an international journal.

3. To agree on research protocols and modelling strategies to ensure that future work is comparable and useful for the LUCIFS objectives.

Copies of the LUCIFS Project Implementation plan (R.J. Wasson ed, 1996 Land Use and Climate Inputs on Fluvial systems During the Period of Agriculture, PAGES Workshop Report, Series 96-2, 51pp) are available from the PAGES International Project Office. The first LUCIFS newsletter, from which the text of this article has been drawn, is available on-line at http://www.pages.unibe.ch/publications/ publications.html.

\section{BoB WAsson}

Geography Department, Australian National University, Canberra 0200, Australia

robert.wasson@anu.edu.au

\section{Second Workshop on Global Paleoenvironmental Data}

\author{
Boulder, CO, USA, February 9-12, 1998
}

Putting individual paleorecords into a multi-proxy, multi-disciplinary, global context is the only way to synthesize a truly global picture of past climatic and environmental change. The difficulties inherent in synthesizing research across-disciplines and between countries are well known, but not insurmountable. Many of the past successes in paleodata management, including the hotoff-the-presses Greenland Ice Core Data CD, Ocean Drilling Program Database, International Tree Ring Data Bank, and North American and European Pollen Databases have understandably been organized independently, primarily along disciplinary and geographical lines. This workshop brought together data management experts from 43 different PAGES data efforts in an attempt to produce an implementation plan for a broad set of guidelines on how to set up a regional or thematic data effort.

One of the principle conclusions of the group was the necessity for making databases consistent in terms of their required documentation and formats. Databases need to be useful to people from outside the field, who may wish to extract information for comparative purposes without excessive complications. At the same time they should serve the needs of scientists who require a detailed accounting of the raw data, any methods used to filter the data and the manner in which climatic or environmental information has been inferred from the data as well as a careful quantification of the uncertainties. It was agreed that the primary responsibility for proper interpretation of archived data lies with the individual users, just as the ultimate quality of the archived data rests with the individual contributors. Nonetheless, effort should be made to structure and maintain databases so as to maximize ease of access and submission and at the same time minimize potential pitfalls of data misinterpretation.

The group took as its starting point the World Data Center System Guide, published by the International Council of Scientific Unions in 1996, and the Global Paleoenvironmental Data report (PAGES report 95-2), which arose out of the first PAGES data workshop held in 1993. Although these documents provide an admirable foundation, the world of data management has blossomed exponentially in the past decade and an update is required. A two part report entitled "The PAGES Data Guide" is being prepared by the 